\title{
Predictors of invasion in needle core biopsies of the breast with ductal carcinoma in situ
}

\author{
Edna May L Go ${ }^{1,2}$, Siu-Ki Chan ${ }^{3}$, Joaquim SL Vong ${ }^{1}$, Philip CW Lui ${ }^{1}$, Anthony WH Chan ${ }^{1}$, \\ Tony KF Ma ${ }^{4}$, Mark A Ang ${ }^{2}$, Bonita KB Law ${ }^{5}$, Puay-Hoon Tan ${ }^{6}$ and Gary M Tse ${ }^{1}$ \\ ${ }^{1}$ Department of Anatomical and Cellular Pathology, Prince of Wales Hospital, The Chinese University \\ of Hong Kong, Hong Kong SAR, China; ${ }^{2}$ Department of Laboratories, University of the Philippines, Manila, \\ Philippines; ${ }^{3}$ Department of Pathology, Kwong Wah Hospital, Hong Kong SAR, China; ${ }^{4}$ Department of \\ Pathology, North District Hospital, Hong Kong SAR, China; ${ }^{5}$ The Breast Center, Union Hospital, Hong Kong \\ $S A R$, China and ${ }^{6}$ Department of Pathology, Singapore General Hospital, Singapore
}

\begin{abstract}
A significant proportion of ductal carcinomas in situ (DCISs) of the breast diagnosed on core biopsies had invasion upon excision. An assessment of various invasion predictors in the biopsies yielded conflicting results. A cohort of 157 cases with needle core biopsy diagnosed with DCISs (including 109 histologically proven DCISs, and 48 cases with invasion upon excision) were evaluated for the numbers of positive and total cores, the percentage of positivity, lobular cancerization, tumor nuclear grade, necrosis, calcification, predominate histological pattern, lymphocytic infiltrate and excisional tumor size. The mean positive core percentage and excisional tumor size were $76 \%$ and $2.8 \mathrm{~cm}$ for invasive and $66 \%$ and $1.9 \mathrm{~cm}$ for noninvasive groups. In the biopsy of the invasive group, cancerization of lobules was present in $\mathbf{5 2} \%$, and nuclear grades $\mathbf{1}$, 2 and 3 were present in 31,31 and $38 \%$, respectively. Large comedo and small noncomedo necroses were present in 48 and $10 \%$, whereas large and small calcifications were present in 16 and $21 \%$. Solid, cribriform and papillary patterns were observed in 88,38 and $21 \%$, respectively. Moderate to marked lymphoid infiltrate was present in $31 \%$. In the biopsy of the noninvasive group, cancerization of lobules was present in $69 \%$, and the nuclear grades 1, 2 and 3 were present in 23,48 and $29 \%$, respectively. Large comedo and small noncomedo necroses were present in 35 and $11 \%$, whereas large and small calcifications were present in 33 and $23 \%$. Solid, cribriform and papillary patterns were observed in 85,39 and $9 \%$, respectively. Moderate to marked lymphoid infiltrate was present in $36 \%$. Comparing these groups, a higher positive core percentage, papillary pattern and less cancerization of lobules in the cores and larger excisional tumor size were associated with a higher chance of invasion. Calcification, necrosis and nuclear grade were not significant invasion predictors.
\end{abstract}

Modern Pathology (2010) 23, 737-742; doi:10.1038/modpathol.2009.187; published online 15 January 2010

Keywords: breast; core biopsy; ductal carcinoma in situ

Percutaneous needle core biopsy of the breast is a well-established technique in the diagnostic workup of breast lesions, with a high accuracy and sensitivity. ${ }^{1-6}$ With the increasing use of needle core biopsy as the initial diagnostic workup in dealing with breast lesions, and the widespread use of mammographic screening resulting in increased detection of asymptomatic calcifications, more and more ductal

Correspondence: Dr GM Tse, FRCPC, Department of Anatomical and Cellular Pathology, Prince of Wales Hospital, Ngan Shing Street, Shatin, Hong Kong SAR, China.

E-mail: garytse@cuhk.edu.hk

Received 12 August 2009; revised 22 October 2009; accepted 23

October 2009; published online 15 January 2010 carcinomas in situ (DCISs) are being detected using needle core biopsy. However, some cases of needle core biopsy-diagnosed DCISs turned out to be invasive in the final excisions. This was not an uncommon occurrence, being reported in $8-44 \%^{7-18}$ of multiple series of needle core biopsy-diagnosed DCISs of the breast. It is interesting to note that the upstaging rates of several series spanning over a period of time were similar, indicating that there has not been substantial progress in the histological evaluation of predictive factors of invasion in needle core biopsy showing DCIS only. In fact, review of the series in the literature gave conflicting results. Many of these series evaluated various factors, but so far no single factor stood out to be a consistent 
predictor. The factors that had been evaluated could be categorized as radiological features (mass, density, radiologic extent as measured using mammography or sonography, and the extent and morphology of the calcifications), ${ }^{8,10,13,14,16,19}$ histological features (size, grade, necrosis, calcifications, architecture, lobular extension, periductal inflammation and periductal stromal fibrosis), ${ }^{7-10,12,14-16,18-21}$ sampling adequacy as indicated by the number of cores sampled ${ }^{16,18}$ and patient factor, such as age. ${ }^{10,16}$ There were reports that either confirmed or refuted their roles as predictors for invasion in needle core biopsy showing DCIS only. Furthermore, all of these series of needle core biopsy-diagnosed DCISs showed significant upstaging at final excision, indicating that understaging was an inherent problem with this mode of investigation. In this study, a large cohort of pure DCISs as diagnosed using needle core biopsy was collected, and the final excision samples were reviewed to identify upstaged cases. Various histological features were assessed on the original core biopsy for identifying any potential histological predictors for invasion detection.

\section{Materials and methods}

The histopathology files of two of the involved institutions were searched for breast needle core biopsy with a diagnosis of DCIS over a period of 10 and 3 years, respectively. Only those cases with subsequent excision of the lesions were included in the study. All the specimens were fixed in $10 \%$ buffered formalin, and were routinely processed and stained with hematoxylin and eosin (H\&E). All the slides of these cases were retrieved, and reviewed by two pathologists, and the diagnosis was confirmed. Any discrepancies were resolved by discussion at a multi-head microscope. In the needle core biopsy slides, if foci of invasions were identified upon review, the case would also be excluded. For each case, the patients' age was documented.

In the needle core biopsy slides, the following parameters were evaluated:

1. The number of cores sampled and number of cores with tumor, irrespective of the percentage of volume of tumor involvement in each core.

2. The percentage of number of positive cores over total number of cores.

3 . The presence of cancerization of lobules, as defined as involvement of one or more ductal lobular units by the typical malignant cells in the DCISs without distension of the involved ductal lobular units.

4. The nuclear grade of the tumor, with grade 1 indicating low nuclear grade with mild pleomorphism and low mitotic count and small nucleoli; grade 2 indicating intermediate nuclear grade; and grade 3 indicating high nuclear grade with highly pleomorphic nuclei with hyperchromasia, multiple nucleoli and irregular nuclear outline.

5. Necrosis, which was scored as either present or absent, irrespective of the extent of the involvement. Necrosis was also divided into noncomedo necrosis or comedo necrosis, with the latter being more extensive, and found associated with high nuclear grade tumor cells.

6. Calcification, which was scored as either present or absent, irrespective of the extent of calcification. Calcifications were associated with the carcinoma cells, and those that were found in the stroma, nontumorous areas and in the blood vessels were excluded.

7. The predominate histological pattern of the DCIS, including either solid, cribriform, papillary or micropapillary.

8. The presence of lymphocytic infiltrate in the stroma around the DCIS. The intensity of the infiltrate was graded into mild, moderate and severe.

The histological slides of the subsequent excision specimens were also reviewed, and the maximal sizes of the excised lesions measured. In addition, the lesions were diagnosed as DCIS only, DCIS with micro-invasion, as defined by the maximal invasive focus being $1 \mathrm{~mm}$, and DCIS with frank invasion, as defined by the size of the largest invasive focus being $>1 \mathrm{~mm}$. The number of invasive foci did not alter the diagnosis.

\section{Statistical Analysis}

Kruskal-Wallis tests were used for comparing the age of patients, number and mean cores, and excisional tumor sizes among different groups. Chi-square tests were performed for comparing the nuclear grade, necrosis, calcification, architectural histotypes and lymphoid infiltrate among various groups. All statistical analyses were carried out by using SPSS version 16.0 for Windows. A two-tailed $P$-value of $<0.05$ was regarded as statistically significant.

\section{Results}

A total of 157 cases were included in this study, derived from 157 patients, with one biopsy from each patient. The overall age range was $30-84$ years (mean 52 years). In all, 84 cases were from the left breast, and 69 were from the right breast. The number of cores ranged from 2 to 21, with a mean of 5.9 cores per case. The number of positive cores ranged from 1 to 14 , with a mean of 3.7 cores per case. The percentage of positive cores ranged from 6.7 to $100 \%$ (mean $69 \%$ ). Within the biopsies, 100 cases showed cancerization of lobules, whereas 57 cases did not. A total of 38 cases were of nuclear grade 1, 71 were of nuclear grade 2 and 48 were of 
Table 1 Comparison of needle core biopsy parameters between cases with eventual micro-invasion or frank invasion

\begin{tabular}{|c|c|c|c|}
\hline & $\begin{array}{l}\text { Micro- } \\
\text { invasive }\end{array}$ & $\begin{array}{l}\text { Frankly } \\
\text { invasive }\end{array}$ & $\mathrm{P}$-value \\
\hline Age, range (vears) & $33-77$ & $36-84$ & - \\
\hline Age, mean (years) & 52 & 54 & NS \\
\hline Number of cores, range & $2-15$ & $2-15$ & - \\
\hline Number of cores, mean & 3.5 & 4.6 & NS \\
\hline Number of positive cores, range & $1-13$ & $1-13$ & - \\
\hline Number of positive cores, mean & 3.4 & 3.4 & NS \\
\hline Percentage of positive cores, range & $20-100$ & $20-100$ & - \\
\hline Percentage of positive cores, mean & 75 & 77 & NS \\
\hline Cancerization of lobules & $12 / 22$ & $13 / 26$ & NS \\
\hline Nuclear grade 1 & 5 & 10 & NS \\
\hline Nuclear grade 2 & 6 & 9 & NS \\
\hline Nuclear grade 3 & 11 & 7 & NS \\
\hline Large necrosis & $12 / 22$ & $11 / 26$ & NS \\
\hline Small necrosis & $2 / 22$ & $1 / 26$ & NS \\
\hline Calcification, negative & 13 & 17 & NS \\
\hline Calcification, large & 3 & 5 & NS \\
\hline Calcification, small & 6 & 4 & NS \\
\hline Solid pattern & 21 & 21 & NS \\
\hline Cribriform pattern & 6 & 12 & NS \\
\hline Papillary pattern & 4 & 6 & NS \\
\hline Micropapillary pattern & 0 & 1 & NS \\
\hline Lymphoid infiltrate, negative & 2 & 12 & 0.005 \\
\hline Lymphoid infiltrate, mild & 9 & 10 & \\
\hline Lymphoid infiltrate, moderate & 4 & 2 & \\
\hline Lymphoid infiltrate, severe & 7 & 2 & \\
\hline
\end{tabular}

NS: not significant.

nuclear grade 3. Large comedo-type necroses were present in 61 cases and were absent in 96 cases. Small foci of noncomedo necroses were present in 15 cases and were absent in 142 cases. Calcification was absent in 80 cases, and large calcifications were present in 47 cases and small calcifications were present in 30 cases. For the various histological architectural types, solid pattern was present in 135 cases, cribriform pattern in 61 cases, papillary pattern in 20 cases and micropapillary pattern in 6 cases. Lymphoid infiltrate was absent in 41 cases, mild in 62 cases, moderate in 31 cases and severe in 23 cases. Review of the excision specimens showed that among all 157 cases, there were 26 frankly invasive cases and 22 cases with micro-invasion. In 109 cases, the excision specimen confirmed the biopsy diagnosis of DCIS without any invasion. In this series, the upgrading rate was 48 of 157 cases $(30.5 \%)$. The overall tumor sizes ranged from 0.15 to $7.5 \mathrm{~cm}$ (mean $2.2 \mathrm{~cm}$ ).

The biopsy details of the 26 frankly invasive and 22 micro-invasive cases are listed in Table 1. In comparing these two groups, the only difference was the presence of more lymphoid infiltrate in the micro-invasive group than the group with frank invasion. There was no difference among all other various parameters assessed in the biopsy.

Combining the frankly invasive and micro-invasive groups to an overall invasive group yielded a total of 48 cases. The biopsy details of this invasive group, as well as the 109 cases in the noninvasive
Table 2 Comparison of needle core biopsy parameters between cases with eventual invasion or noninvasion

\begin{tabular}{lccc}
\hline & $\begin{array}{c}\text { All- } \\
\text { invasive }\end{array}$ & $\begin{array}{c}\text { Non- } \\
\text { invasive }\end{array}$ & P-value \\
\hline Age, range (years) & $33-84$ & $30-81$ & - \\
Age, mean (years) & 53 & 51 & $\mathrm{NS}$ \\
Number of cores, range & $2-15$ & $2-21$ & - \\
Number of cores, mean & 4.9 & 6.4 & $\mathrm{NS}$ \\
Number of positive cores, range & $1-13$ & $1-14$ & - \\
Number of positive cores, mean & 5.4 & 3.7 & $\mathrm{NS}$ \\
Percentage of positive cores, range & $20-100$ & $6.7-100$ & - \\
Percentage of positive cores, mean & 76 & 66 & 0.039 \\
Cancerization of lobules & $25 / 48$ & $75 / 109$ & 0.045 \\
Nuclear grade 1 & 15 & 25 & $\mathrm{NS}$ \\
Nuclear grade 2 & 15 & 52 & $\mathrm{NS}$ \\
Nuclear grade 3 & 18 & 32 & $\mathrm{NS}$ \\
Large necrosis & $23 / 48$ & $38 / 109$ & $\mathrm{NS}$ \\
Small necrosis & $3 / 48$ & $12 / 109$ & $\mathrm{NS}$ \\
Calcification, negative & 30 & 50 & $\mathrm{NS}$ \\
Calcification, large & 8 & 36 & $\mathrm{NS}$ \\
Calcification, small & 10 & 23 & NS \\
Solid pattern & 42 & 93 & $\mathrm{NS}$ \\
Cribriform pattern & 18 & 43 & $\mathrm{NS}$ \\
Papillary pattern & 10 & 10 & 0.044 \\
Micropapillary pattern & 1 & 5 & NS \\
Lymphoid infiltrate, negative & 14 & 27 & NS \\
Lymphoid infiltrate, mild & 19 & 43 & NS \\
Lymphoid infiltrate, moderate & 6 & 25 & NS \\
Lymphoid infiltrate, severe & 9 & 14 & NS \\
Excision size, range (cm) & $0.3-6$ & $0.15-7.5$ & - \\
Excision size, mean (cm) & 2.8 & 1.9 & 0.044 \\
& & &
\end{tabular}

NS: not significant.

group, are listed in Table 2. Comparing these overall invasive and noninvasive groups, the percentage of positive cores showed a significant difference between the two groups, with a higher percentage noted in the invasive group $(75.9 \%)$ than the noninvasive group $(66.1 \% ; P=0.039)$. In the core biopsies, a papillary pattern is associated with higher incidence of upgrading in the excision specimen ( $9 \%$ in noninvasive group, and $21 \%$ in invasive group, $P=0.044$ ). In addition, cancerization of lobules were significantly more common in the noninvasive group $(69 \%)$ when compared with the invasive group $(52 \% ; P=0.045)$. The excision lesional sizes were significantly larger in the invasive group compared with the noninvasive group $(P=0.044)$. There were no differences between the invasive and noninvasive groups for other parameters of nuclear grade, necrosis, calcification and lymphocytic infiltrate.

\section{Discussion}

The high accuracy in needle core biopsy diagnosis of breast lesions has resulted in the widespread use of this modality in the pre-treatment diagnosis of many breast lesions; however, there are still specific areas in which diagnostic problems arise. These areas include the differentiation of fibroepithelial 
lesions (fibroadenoma and phyllodes tumor), ${ }^{22-24}$ benign and malignant papillary lesions, ${ }^{25,26}$ as well as the problem of upgrading of diagnosis of needle core biopsy showing DCIS only. Similar to this last problem is the diagnosis of atypical duct hyperplasia in a core biopsy with subsequent upgrade to DCIS. ${ }^{11,27-29}$ These are all problematic because the differentiation is based on either the size or extensiveness of the lesion; and in a core biopsy, sampling error and size estimation is a built-in limitation. These are significant as the treatment methods differ between the different differentials. In the setting of needle core biopsy diagnosis of DCIS, the eventual upgrading percentage ranged from 8 to $44 \%$, and this had a significant effect on the management decision.

In this study, a cohort of needle core biopsydiagnosed DCISs were evaluated to analyze predictive histological factors for the presence of invasion in the final excision diagnosis.

The percentage of positive cores has been shown to be a significant predictor of invasion in the final excision. A higher percentage core is associated with increased incidence of invasion. This may reflect more extensive disease that may be associated with higher chance of invasion, as has been reported by others, citing various cutoff points, including involvement of more than 15 ducts spaces, ${ }^{15}$ or various lesional sizes, ranging from 50 to $15 \mathrm{~mm} .^{7,17,21}$ The advantage of assessing the percentage of positive cores over size estimation or the number of positive cores is that a positive ratio is independent of the core sizes and the total number of cores obtained. It has been previously reported that inadequate (or smaller number)cores was associated with a higher upgrading rate, ${ }^{13,18}$ although the latter study ${ }^{18}$ also showed that core size was not related to missed invasion in the final excision. Whereas the issue of number of cores has not been well documented in this context, it has also been reported to be unrelated to missed invasion. ${ }^{16,21}$ It seems that evaluating the percentage of positive cores is more representative than merely the number of cores taken, as the former would yield a more accurate indication of the extensiveness of the lesion. In this context, one may suggest that size estimation may also be a good parameter to evaluate, as evident by the number of studies evaluating the size either pathologically ${ }^{7,15,17,21}$ or radiologically, either using mammography $y^{7,10,20}$ or ultrasound. ${ }^{19}$ This study also showed the positive association of excisional pathologic lesional size and the presence of missed invasion. However, this is not unanimous. In this study there was no association between the excisional tumor size and invasion. Several studies also indicated that palpability, ${ }^{9,10,18}$ radiological size $^{16}$ and the size of largest tumor focus ${ }^{12}$ were not predictive of missed invasion. One major problem concerning size estimation and palpability is that many of the screen-detected DCISs are asymptomatic and without a mass lesion, and hence nonpalpable.
The architectural histotype has also been shown to be significant, with a papillary pattern being more associated with invasive focus in the excision, whereas other patterns did not show any association. The assessment of histological architectural subtypes has only been rarely assessed in previous studies. Cribriform and papillary pattern associated with comedo necrosis in the biopsy has been previously reported as a predictor for invasion, ${ }^{12}$ whereas another study did not find any significant difference between cribriform and noncribriform pattern observed in the biopsies. ${ }^{21}$ In this series, papillary histotype was identified as an independent predictor for invasion, independent of the presence of comedo necrosis or calcification. This observation may be attributed, in part or totally, by the recent proposal that at least some types of papillary DCIS, particularly the encysted variant or even the solid papillary variants, may in fact be a nodular invasive lesion rather than true in situ lesion, as evidenced by the absence of myoepithelial cell layer around the papillary carcinoma. ${ }^{30,31}$ Furthermore, there is also recent evidence that even the myoepithelial cells that are present around DCIS showed phenotypic alternations when compared with those lining normal ductal lobular units, and this may also have a role in the invasiveness of DCIS. $^{32}$

The current series also showed the absence of cancerization of the lobules by the DCIS in the biopsy to be another predictor for invasion. Lobular extension has been shown to be a significant predictor for invasion, ${ }^{21}$ and when the size is $>0.4 \mathrm{~cm} .{ }^{12}$ In most other studies in the literature, this issue had not been addressed. In the former study, ${ }^{21}$ the ratio of cancerization of lobules in those eventually turned out to be invasive to the noninvasive ones was approximately 2 to 1 , whereas in the latter series ${ }^{12}$ the ratio was approximately 2.9 to 1 . However in this latter study, the criterion of lobular extension was associated with a large aggregate mass; whether lobular extension alone was a significant predictor for invasion had not been confirmed. In the current series, the ratio of cancerization in those that were invasive to noninvasive was 0.76 to 1 . Given the paucity of strong evidence regarding the role of cancerization of lobules as an invasive predictor, this would require further evaluation.

Lymphocytic infiltrate around the tumors in the biopsy has been reported to be associated with increased $^{14}$ or not increased ${ }^{21}$ risk of invasion in the excision. Most other studies did not address this issue. The observation in this series was that a higher intensity of lymphocytic infiltrate was associated with micro-invasion over frank invasion, but the significance was lost when both invasive groups were combined to compare with the noninvasive group, probably suggesting that this observation may be fortuitous. Again, further assessment is warranted. 
Other important findings in this study were the identification of some factors that were probably insignificant in the prediction of invasion for needle core biopsy showing DCIS only. These included patients' age, number of cores taken, nuclear grade, necrosis including both large comedo and small noncomedo types, calcification including large calcification that were usually associated with comedo necrosis, or small calcification, and architectural histotypes other than papillary. Many of these were the cardinal features involved in the grading of the DCIS, including nuclear grade, necrosis and architectural histotypes, whereas others were clinical and radiological parameters, including patients' age, number of cores taken and the presence and size (and morphology) of the calcification. Whether these factors are totally insignificant is still debatable, as all of these had been shown to be significant or insignificant in different series. For instance, some studies showed that age, ${ }^{16}$ number of cores, ${ }^{16,21}$ grade, $^{7-9,11,16,21}$ architecture, ${ }^{7,16,21}$ necrosis, ${ }^{9,11,16,21}$ periductal stromal fibrosis or desmoplasia ${ }^{14-16,21}$ and calcification ${ }^{16,18}$ were insignificant, whereas other studies showed the same factors, including number of cores, ${ }^{13,18}$ grade, ${ }^{10,14,15,20}$ comedo necrosis, ${ }^{10,11,19}$ and periductal inflammatory cells infiltrate ${ }^{14}$ to be significant.

It seems that prediction of eventual invasion in needle core biopsy showing only DCIS is still fraught with uncertainty. We have shown that probable significant predictors for invasion in needle core biopsy included the percentage of positive cores, and an architectural histotype of papillary DCIS. Factors such as patients' age, number of cores taken, the grade, necrosis, calcification and periductal lymphocytic infiltrate were shown in this series to be insignificant. Many of these factors had been shown by some researchers, although not unanimously, to be significant, and there is not enough convincing evidence so far to draw a conclusion. At this stage it is advisable to include all these controversial factors in the biopsy report, particularly including a comment on whether the histotype is papillary or nonpapillary, and also on the percentage of positive cores.

\section{Disclosure/conflict of interest}

The authors declare no conflict of interest.

\section{References}

1 Parker SH, Burbank F, Jackman RJ, et al. Percutaneous large-core breast biopsy: a multi-institutional study. Radiology 1994;193:359-364.

2 Parker SH, Jobe WE, Dennis MA, et al. US-guided automated large-core breast biopsy. Radiology 1993; 187:507-511.

3 Parker SH, Lovin JD, Jobe WE, et al. Stereotactic breast biopsy with a biopsy gun. Radiology 1990;176:741-747.
4 Liberman L, Dershaw DD, Rosen PP, et al. Stereotaxic core biopsy of impalpable spiculated breast masses. AJR Am J Roentgenol 1995;165:551-554.

5 Burbank F. Stereotactic breast biopsy of atypical ductal hyperplasia and ductal carcinoma in situ lesions: improved accuracy with directional, vacuum-assisted biopsy. Radiology 1997;202:843-847.

6 Ciatto S, Houssami N, Ambrogetti D, et al. Accuracy and underestimation of malignancy of breast core needle biopsy: the Florence experience of over 4000 consecutive biopsies. Breast Cancer Res Treat 2007;101:291-297.

7 Dillon MF, McDermott EW, Quinn CM, et al. Predictors of invasive disease in breast cancer when core biopsy demonstrates DCIS only. J Surg Oncol 2006;93: 559-563.

8 Wahedna Y, Evans AJ, Pinder SE, et al. Mammographic size of ductal carcinoma in situ does not predict the presence of an invasive focus. Eur J Cancer 2001;37:459-462.

9 Mittendorf EA, Arciero CA, Gutchell V, et al. Core biopsy diagnosis of ductal carcinoma in situ: an indication for sentinel lymph node biopsy. Curr Surg 2005;62:253-257.

10 Yen TW, Hunt KK, Ross MI, et al. Predictors of invasive breast cancer in patients with an initial diagnosis of ductal carcinoma in situ: a guide to selective use of sentinel lymph node biopsy in management of ductal carcinoma in situ. J Am Coll Surg 2005;200:516-526.

11 Méndez I, Andreu FJ, Sáez E, et al. Ductal carcinoma in situ and atypical ductal hyperplasia of the breast diagnosed at stereotactic core biopsy. Breast J 2001;7:14-18.

12 Renshaw AA. Predicting invasion in the excision specimen from breast core needle biopsy specimens with only ductal carcinoma in situ. Arch Pathol Lab Med 2002;126:39-41.

13 Jackman RJ, Burbank F, Parker SH, et al. Stereotactic breast biopsy of nonpalpable lesions: determinants of ductal carcinoma in situ underestimation rates. Radiology 2001;218:497-502.

14 Hoorntje LE, Schipper ME, Peeters PH, et al. The finding of invasive cancer after a preoperative diagnosis of ductal carcinoma-in-situ: causes of ductal carcinoma-in-situ underestimates with stereotactic 14-gauge needle biopsy. Ann Surg Oncol 2003;10: 748-753.

15 Bonnett M, Wallis T, Rossmann M, et al. Histologic and radiographic analysis of ductal carcinoma in situ diagnosed using stereotactic incisional core breast biopsy. Mod Pathol 2002;15:95-101.

16 Lee CH, Carter D, Philpotts LE, et al. Ductal carcinoma in situ diagnosed with stereotactic core needle biopsy: can invasion be predicted? Radiology 2000;217: $466-470$.

17 Lagios MD, Westdahl PR, Margolin FR, et al. Duct carcinoma in situ. Relationship of extent of noninvasive disease to the frequency of occult invasion, multicentricity, lymph node metastases, and shortterm treatment failures. Cancer 1982;50:1309-1314.

18 Rutstein LA, Johnson RR, Poller WR, et al. Predictors of residual invasive disease after core needle biopsy diagnosis of ductal carcinoma in situ. Breast J 2007;13:251-257.

19 Leikola J, Heikkilä P, Pamilo M, et al. Predicting invasion in patients with DCIS in the preoperative percutaneous biopsy. Acta Oncol 2007;46:798-802. 
20 Meijnen P, Oldenburg HS, Loo CE, et al. Risk of invasion and axillary lymph node metastasis in ductal carcinoma in situ diagnosed by core-needle biopsy. Br J Surg 2007;94:952-956.

21 Huo L, Sneige N, Hunt KK, et al. Predictors of invasion in patients with core-needle biopsy-diagnosed ductal carcinoma in situ and recommendations for a selective approach to sentinel lymph node biopsy in ductal carcinoma in situ. Cancer 2006;107: 1760-1768.

22 Lee AH, Hodi Z, Ellis IO, et al. Histological features useful in the distinction of phyllodes tumour and fibroadenoma on needle core biopsy of the breast. Histopathology 2007;51:336-344.

23 Jacobs TW, Chen YY, Guinee Jr DG, et al. Fibroepithelial lesions with cellular stroma on breast core needle biopsy: are there predictors of outcome on surgical excision? Am J Clin Pathol 2005;124: $342-354$.

24 Lee AH, Hodi Z, Ellis IO, et al. Histological features useful in the distinction of phyllodes tumour and fibroadenoma on needle core biopsy of the breast. Histopathology 2007;51:336-344.

25 Collins LC, Schnitt SJ. Papillary lesions of the breast: selected diagnostic and management issues. Histopathology 2008;52:20-29.
26 Tse GM, Tan PH, Lacambra MD, et al. Papillary lesions of the breast - accuracy of core biopsy. Histopathology (in press).

27 Ely KA, Carter BA, Jensen RA, et al. Core biopsy of the breast with atypical ductal hyperplasia: a probabilistic approach to reporting. Am J Surg Pathol 2001;25:1017-1021.

28 Meyer JE, Smith DN, Lester SC, et al. Large-core needle biopsy of nonpalpable breast lesions. JAMA 1999;281: 1638-1641.

29 Houssami N, Ciatto S, Ellis I, et al. Underestimation of malignancy of breast core-needle biopsy: concepts and precise overall and category-specific estimates. Cancer 2007;109:487-495.

30 Collins LC, Carlo VP, Hwang $\mathrm{H}$, et al. Intracystic papillary carcinomas of the breast: a reevaluation using a panel of myoepithelial cell markers. Am J Surg Pathol 2006;30:1002-1007.

31 Hill CB, Yeh IT. Myoepithelial cell staining patterns of papillary breast lesions: from intraductal papillomas to invasive papillary carcinomas. Am J Clin Pathol 2005;123:36-44.

32 Hilson JB, Schnitt SJ, Collins LC. Phenotypic alterations in ductal carcinoma in situ-associated myoepithelial cells: biologic and diagnostic implications. Am J Surg Pathol 2009;33:227-232. 